\title{
Exploring the relationship between loneliness and social cognition in older age
}

Martina De Lillo, Andrew Martin and Heather J. Ferguson*

University of Kent, U.K.

Corresponding author:

Heather Ferguson (h.ferguson@kent.ac.uk; iD https://orcid.org/0000-0002-1575-4820)

Article submitted for consideration to the Special Issue, "Understanding Others in Moments of Crisis" in Social Psychology.

\section{Acknowledgements}

This work was carried out with the support of a European Research Council grant to HJF (Ref: CogSoCoAGE; 636458).

Word count: 7,939 


\begin{abstract}
Understanding others is a key component of successful social interactions, and declines in social abilities during later life can lead to social isolation and loneliness. We investigated the relationship between different sub-components of social cognition and loneliness in a large sample of older adults. We tested perspective-taking and mentalizing skills, alongside selfreported loneliness and social functioning. Results revealed a significant effect of loneliness on older adults' ability to resist egocentric interference when taking others' perspectives. However, this effect was eliminated when age was added to models, which suggests that egocentric tendencies increase with age, and people experience increasing levels of loneliness and feelings of social isolation with increasing age. Mentalizing and interference from others' perspectives were not influenced by loneliness or age.
\end{abstract}




\section{Introduction}

Human social interactions are complex; they involve diverse social cognitive processes, such as taking other peoples' perspectives and representing their mental states (e.g., desires, beliefs). These abilities fall under the general concept of Theory of Mind (ToM; Wimmer \& Perner, 1983) since ToM includes the ability to understand and infer others' mental states. Previous literature has shown how these abilities change across the lifespan. ToM develops during early childhood (Leslie, 2005; Devine \& Hughes, 2013) and adolescence (Dumontheil et al., 2010; Symeonidou et al., 2020), and declines in older age (Henry et al., 2013; Maylor et al., 2002). While a significant amount of research explored the early development of ToM, interest in examining social communication skills in later adulthood has emerged over the last couple of decades, since impairments in these skills can have significant negative consequences in everyday life. This study aims to shed light on some of the factors that influence ToM abilities in an aging population.

Older adults experience difficulty in understanding others' mental states in false belief tasks (Bernstein et al., 2011; Bradford et al., submitted), faux paus (Cavallini et al., 2013; Halberstadt et al., 2011), and irony (Phillips et al., 2015), especially in complex scenarios (Lecce et al., 2019). In particular, older adults show a bias to anchor understanding to their own egocentric perspective (Bradford et al., submitted; Saryazdi \& Chambers, 2020; Mattan et al., 2017), and experience difficulty in switching between their own and another person's perspective (Martin et al., 2019). In turn, research has shown that these age-related difficulties in ToM mediate a substantial decline in social participation in older adults, which in turn leads to isolation, loneliness and poor health (Bailey et al., 2008; Berkman, 1995; Lund et al., 2018; Santamaría-García, et al., 2020; Cacioppo \& Hawkley, 2009). In this paper 
we examine whether social cognitive skills in older age are affected by subjective feelings of loneliness and social isolation.

It is known that the number and quality of social relationships can affect ToM processes. People who have a higher number of meaningful relationships are more likely to make social inferences about others' mental states, such as their thoughts and intentions (Stiller \& Dunbar, 2007), and people who feel higher levels of loneliness report a greater difficulty in social functioning (Tan et al., 2020). Loneliness has been found to be positively correlated with age, and increases further when social networks are reduced (Pinquart \& Sorensen, 2001). Lecce et al. (2017) found that differences in ToM performance in older adults is linked to having friendships, which confirms the importance of qualitative meaningful relationships in older people. Similarly, the size of one's social network and the level of sociability and participation in group activities moderates the relationship between age and social judgements (Hess et al., 2005). Interestingly, the reverse relationship has also been observed, as lower performance on tasks that measure social cognition significantly predicts higher levels of loneliness (Trémeau et al., 2016). Moreover, difficulties with social cognition can impact on well-being and our relationships (Spitzberg and Hurt, 1987 Tsai and Reis, 2009); social relations are crucial for health, especially in older age (Diener \& Seligman, 2002; Matheson, 2011; Shankar et al., 2014). Thus, a reduced social network and experiences of maladaptive social interactions due to impaired social understanding can lead to isolation, and consequently, to loneliness.

Loneliness is defined as a negative emotion generated by the conflicted wished and actual quality of social relations (Hawkley \& Cacioppo, 2010), loneliness is typically associated with a diminished number of friends, as well as a low evaluation of the quality of 
interpersonal relationships (Nangle et al., 2003). Loneliness seems to enhance the severity of psychiatric disorders (Lim \& Gleeson, 2014; Lim et al., 2018; Meltzer et al., 2013; Trémeau et al., 2016) and mental ill-health (Cacioppo et al. 2006; Domènech-Abella et al., 2017; Lim, et al., 2016). Moreover, people who experience loneliness are more vulnerable to cognitive decline (Boss et al., 2015; Tilvis et al., 2004) and Alzheimer's disease (Wilson et al., 2007).

Older adults are especially susceptible to loneliness since they are more likely to experience life events that reduce their social network and lead to social isolation, such as retirement, the loss of a partner, or relatives moving away. Due to poor physical health and/or reduced mobility, older people can experience reduced opportunities to join social events and engage in social interaction, which leads to detrimental effects on ToM. In the last two years, the COVID-19 pandemic has had a significant impact on our social lives, forcing many individuals to withdraw from their normal social life, which has consequences on their physical and psychological health (e.g. Hawkley \& Capitanio, 2015; Valtorta et al., 2016). This impact is particularly evident among older people. Very recent aging studies have highlighted that older adults report higher levels of social isolation (Peng \& Roth, 2021), reduction of social relationships (Heid et al., 2021) and loneliness (Carson et al., 2020; Heidinger et al., 2021) during the global pandemic. It is therefore timely to better understand how social cognitive processing is influenced by high levels of loneliness and feelings of isolation in older age, which represent 'moments of crises'.

Despite the growing interest in understanding how social cognitive processing changes in older age, and why loneliness increases in later life, very little research has empirically tested the relationship between loneliness and ToM in older age. In the current study, we address this gap in the literature by adopting well established paradigms to examine the relationship 
between different social cognitive abilities and loneliness in a sample of older adults.

Perceived loneliness was assessed using the Revised UCLA Loneliness Scale (Russell, 1996), and a self-report measure captured individuals social functioning, since it characterizes social interaction and has been shown to decline in older age (Berkman et al., 2000). The current study is distinct from previous studies because it examines the effect of age within a diverse older group (aged 60 to 86 years old), rather than comparing extreme young and old groups, and employs multiple tasks to capture different sub-components of ToM using continuous measures (i.e. avoiding ceiling effects). In particular, we focused on two sub-components of ToM that are known to be susceptible to age-related decline: perspective-taking and mentalizing abilities.

To examine perspective-taking skills we adopted the 'director' interactive reference assignment task and the 'dot-probe' visual perspective-taking task. In the interactive reference assignment task, participants follow the instructions on a director to move objects around a gridded cupboard. Importantly, some of the objects are occluded from the director's view, thus participants need to infer their visual perspective to identify the intended target object. Research using this task has shown that participants are susceptible to egocentric biases, and frequently select the perspective inappropriate object because it fits their own privileged view. Moreover, it has been shown that older adults commit more egocentric errors than younger adults in this task and are more likely to consider competitor objects in privileged view (Bradford et al., submitted; Hultsch et al., 2002; Saryazdi \& Chambers, 2020). In the visual perspective-taking task (Samson et al., 2010), participants make judgements about what can be seen either from their own or an avatar's perspective (Level 1 perspective-taking; Flavell et al. 1986), when these two perspectives are either consistent or in conflict. Participants experience two kinds of interference in this task: from their own 
visual perspective when judging the avatar's perspective (egocentric interference), and from the avatar's visual perspective when judging their own perspective (altercentric interference). Using a version of this task, previous research has shown that older adults exhibit a preference for self-relevant perspectives, and experience a particular difficulty in switching between their own and the avatars' perspective (Mattan et al., 2017; Martin et al., 2019). These previous studies therefore suggest that difficulties with perspective-taking in older age are due to specific impairments in the cognitive control mechanisms that manage egocentric biases.

To assess metalizing abilities we adopted the Strange Stories task (White et al., 2009), in which participants answer questions about the mental states of characters in short narratives. There have been mixed findings with this task in older age, with one study showing enhanced performance among older compared to young adults when inferring mental states (Happé et al., 1998), and another reporting a decline that begins around 30 years old (Brunsdon et al., 2019). By using this task, we hoped to provide further insights on how mentalizing is affected in older age, and the social context factors that might influence this. To summarize, we adopted these three tasks because they each tap different sub-components of social cognitive abilities. They have also been used widely in previous aging literature, with results suggesting a decline in performance with advancing age. Finally, all the adopted tasks, examine social cognition on continuous measures and not binary (e.g. true/false) responses, which are often subject to ceiling effects among adults.

In line with previous literature, we predicted that performance on all three social cognition tasks would correlate with age, as participants would make more egocentric responses and be less accurate when inferring others' mental states with increasing age (as seen in Bradford et 
al., submitted; Brunsdon et al., 2019; Hultsch et al., 2002; Mattan et al., 2017; Martin et al., 2019; Saryazdi \& Chambers, 2020). We also expected that increasing age would be associated with reduced self-reported social functioning (Carson et al., 2020; Heidinger et al., 2021; Peng \& Roth, 2021; Heid et al., 2021). More importantly, we hypothesised that selfreported feelings of loneliness would predict performance on the three social cognitive tasks, as well as self-report scores in the social functioning questionnaire, and tested whether the effect of loneliness was distinct for perspective-taking and mentalizing sub-components.

\section{Methods}

\subsection{Participants}

102 participants, aged between 60 and 86 years old, were recruited from the local community through the University of Kent research participation database, newspaper adverts, and social media. All participants were native English speakers, had normal or corrected-to-normal vision, no history of neurological disorders, and had no current psychiatric condition. From this sample, four participants were excluded due to missing data in three or more of the measures (all other participants had full data for all measures). This resulted in a final sample of 98 participants $\left(M_{\text {age }}=70.2 ; s d_{\text {age }}=6.8 ; 66\right.$ females $)$.

\subsection{Measures}

\subsubsection{Interactive Reference Assignment task}

To examine participants' ability to infer reference in conversation we used an avatar version of the referential communication 'Director' task (Keysar et al., 2003). In this task, participants were presented with an image of a room containing a 4 x 4 gridded cupboard, creating 16 slots that could contain objects, and a female avatar (the 'director') standing to the rear right-hand side of the cupboard (see Figure 1). Crucially, the backs of five slots 
(different for each trial) were covered with a green backing, so that only the participant could see the contents of these spaces, and the contents were occluded from the director's view. Eight objects were randomly placed within the grid slots, two of which were in occluded positions and six could be seen by both the director and the participant. Participants were asked to move objects around the grid following the avatar's verbal instructions.

In the Listener perspective condition, participants needed to take the director's perspective to select the mutually available object, and ignore a competitor object in an occluded slot that fitted the description (since it could not be seen by the director). Thus, participants were required to inhibit their own perspective to consider the correct object from the director's point of view. For instance, the participant could be asked to 'Move the small star one slot down', where the grid contained three stars of different sizes, the smallest of which was occluded from the director. In this example, it would be correct for the participant to select the medium sized star, since this is the smallest star from the director's perspective. In the Shared perspective condition, the competitor object was replaced by a different (neutral) object that could not be mistaken for the object in the director's instruction. For instance, the participant could be asked to 'Move the small star one slot down', where the grid contained only two mutually-available stars. Here, it would be correct to select the objectively smallest star, since this matched both the participant's and the director's perspective. Participants responded using the computer mouse to select an object, and drag it to the new location detailed in the verbal instruction. See Figure 1 for a visual depiction of stimuli across Listener and Shared perspective conditions.

Before starting the experiment, participants were given standardized instructions emphasising that the director had a different perspective of the grid than that of the participant; they were 
shown an example stimulus, including viewing the shelves from the participant and the director's perspectives (i.e. with the occluded slots blocking view of the objects inside of them from the director's perspective), to ensure all participants understood that the director could not see all the objects. They were then familiarized with the procedure by completing two practice trials (i.e. six verbal instructions), and received feedback on their responses (practice trials only). The experimenter was present during this phase to ensure that the participant understood the task and to clarify where needed. The main experiment included 24 experimental trials (each with different sets of objects), of which 12 included a Listener perspective instruction and 12 included a Shared perspective instruction. Each trial included three instructions; two were fillers that referred to a specific item and did not involve perspective-taking (e.g., one used a modified description, e.g., 'the yellow bucket', and one used a simple description, e.g., 'the shoe'). Filler instructions were not included in the analysis. The order of filler and critical instructions was counterbalanced across trials, and a new instruction was only given once participants had responded to the previous instruction. Audio instructions were presented through headphones, and participants were given $4000 \mathrm{~ms}$ before the first instruction to inspect the grid. This task lasted 10 minutes on average. Accuracy was calculated as the proportion of trials on which participants correctly selected the target object in the Listener perspective condition (in our sample $M_{a c c}=.82, \mathrm{SD}=.28$ ). 


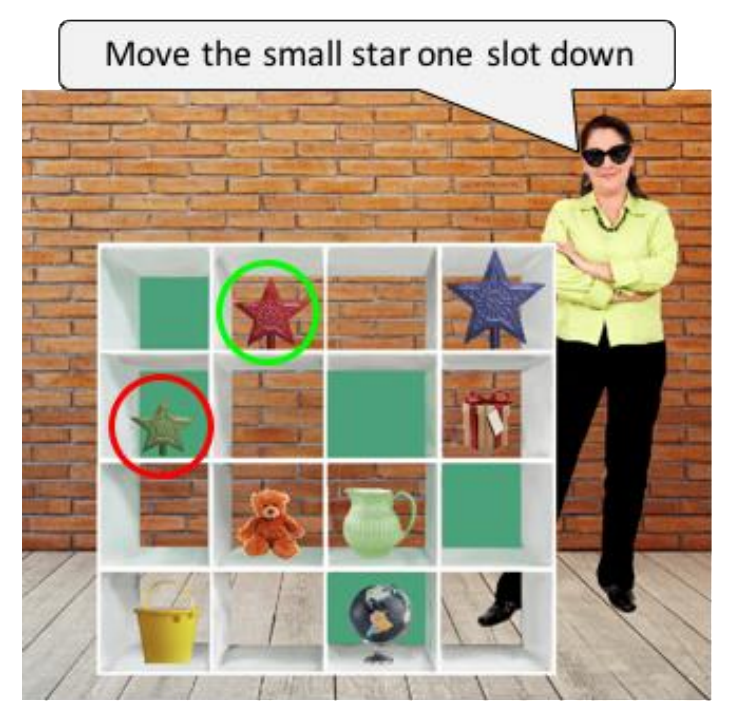

LISTENER PERSPECTIVE

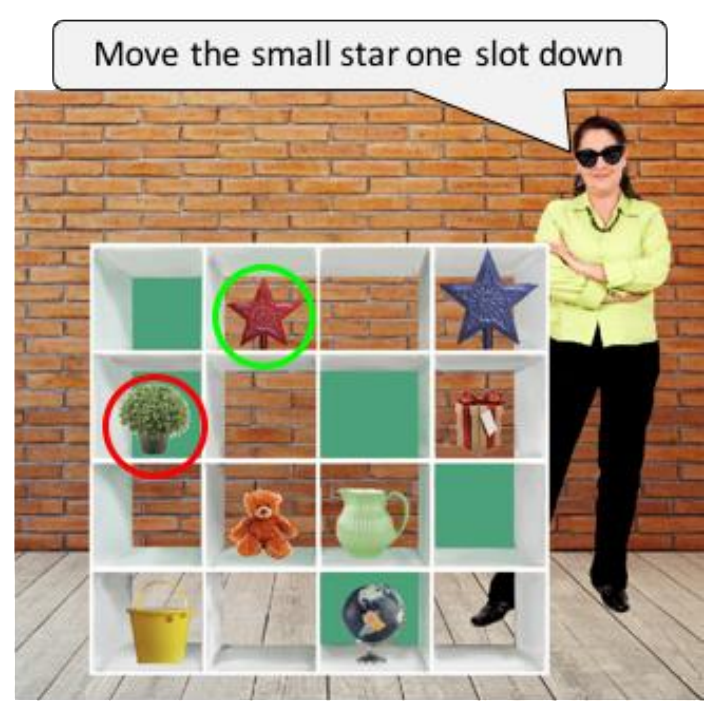

SHARED PERSPECTIVE

Figure 1: An example of the stimuli used in the Interactive reference assignment task, showing Listener perspective and Shared perspective conditions.

\subsubsection{Strange Stories task}

We adopted the computerised task from White et al. (2009). Participants were presented (visually and auditorily) with 24 stories about everyday events, then had to respond verbally to questions that required them to infer peoples' mental states, their physical actions, or physical events in nature (eight of each type). Participants' answers were recorded and scored using White et al. (2009)'s criteria, and a 'ToM score' was calculated from the human mental state stories. The maximum score on this measure was 16 , indicating high proficiency in ToM (in our sample $M=13.3, \mathrm{SD}=2.2$ ). The task took approximately 15 minutes to complete.

\subsubsection{Visual perspective-taking task}

Visual perspective-taking was assessed using the 'dot-probe' task (Ferguson et al., 2017; Samson et al., 2010), and was controlled using Experiment Builder software. Participants were asked to verify the number of discs that were visible from either their own perspective 
(self-perspective condition) or according to an avatar's perspective (other perspective condition). Each trial began with a fixation point, a blank screen $(500 \mathrm{~ms})$, a perspective cue (750ms; 'YOU' or 'SHE/HE'), a second blank screen (500ms), a number cue (750ms; 0, 1, 2, $3)$, followed by the target image of a room ( $650 \times 480$ pixels). This target image showed a lateral view of a room with left, back and right walls. Red discs were placed on either or both left and right walls. An avatar was positioned in the centre of the room and on half of trials was facing the left wall, and half of the trials was facing the right wall. Female participants were presented with a female avatar and male participants were presented with a male avatar. On half of the trials, the avatar could see the same number of discs as the participant (consistent condition) and on the other half of the trials the avatar could not see all the discs that the participant could see (inconsistent condition). Participants pressed a green button if the number cue matched the number of discs visible from the cued perspective and a red button if the number cue mismatched the number of discs visible from either the self or other perspective. Trials ended with a button press or after $3000 \mathrm{~ms}$. The trial sequence is displayed in Figure 2.
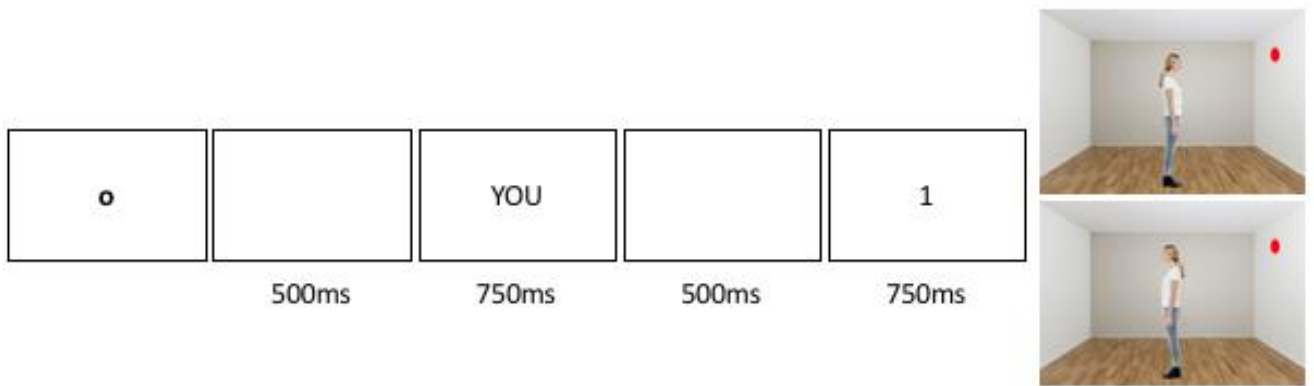

Self Consistent

Self Inconsistent

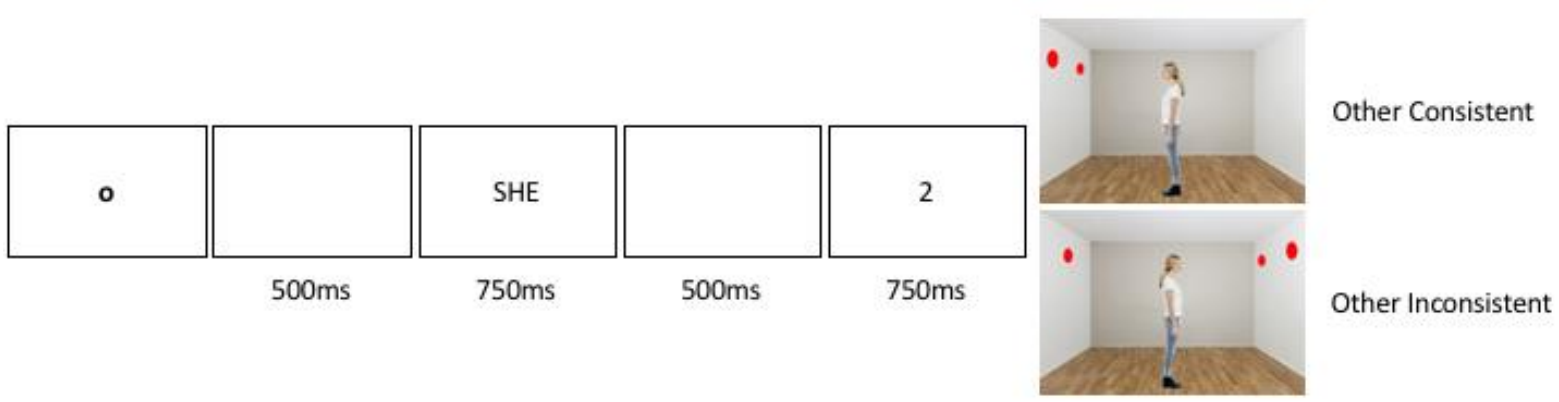


Figure 2: Schematic trial sequence and visual displays presented to participants in the visual perspective-taking task.

Participants completed 23 practice trials (with feedback), followed by four experimental blocks, with 52 trials per block and a self-directed break in-between blocks. Overall, there were 96 matching trials, 96 mismatching trials, and 16 "filler" trials in which no discs were displayed on either wall. There were 48 trials in the self-perspective condition and 48 trials in the other perspective condition, of which half were in the consistent condition and half were in the inconsistent condition. Trials were presented in a pseudo-randomised order so that trials were equally likely to have been preceded by a same perspective trial as a different perspective trial. The maximum number of consecutive trials that tapped the same perspective was four, and there were no more than three consecutive trials of the same perspective-consistency condition. No complete stimulus repetitions (i.e. same perspective cue and image) were included. The task took approximately 25 minutes to complete.

Following Samson et al. (2010), only matching trials were included in the analysis (i.e. trials where the number cue matched the number of discs visible from the cued perspective). We recorded response times for correct responses within 3000ms of image onset, removed individual trial outliers that fell more than 2.5SDs of each participant's mean, and used these to calculate an egocentric bias score (inconsistent minus consistent for 'other' perspective trials; in our sample $M=189 \mathrm{~ms}, \mathrm{SD}=202$ ) and an altercentric bias score (inconsistent minus consistent for 'self' perspective trials; in our sample $M=101 \mathrm{~ms}, \mathrm{SD}=163$ ), with higher scores in each indicating greater interference from the alternative perspective. 


\subsubsection{Revised UCLA Loneliness Scale}

The Revised UCLA Loneliness Scale (Russell, 1996: R-UCLA) was used to measure participants' subjective level of loneliness and feelings of social isolation. Participants were given 20 statements, such as 'I lack companionship', and responded to each using a 4-point scale from 'Never' to 'Often'. The scale produced a maximum score of 80 (indicating high levels of perceived loneliness; in our sample $M=35.2, \mathrm{SD}=9.6$ ).

\subsubsection{Social Functioning Scale}

The Social Functioning Scale (Birchwood et al., 1990) was used as a self-report measure of general social functioning. It consists of seven sub-scales, however only three were administered in the current study: (1) Social Engagement/Withdrawal (e.g., time spent alone/initiation of conversation; 'How many hours of the waking day do you usually spend alone?'), (2) Interpersonal Behaviour (e.g., number of close friends, quality of communication; 'How easy or difficult do you find it talking to people at the moment?), and (3) Prosocial Activities (e.g., how often they participated in different social activities, such as cinema, sports or parties, over the last three months). The sum of the three sub-scale scores was used as the self-reported social functioning measure (maximum possible score was 90), with higher scores representing a better social functioning level and frequency of behaviour (in our sample $M=51.7, \mathrm{SD}=9.7$ ).

\section{Results}

All analyses were conducted in R version 3.6.1 (R Core Team, 2019). To test for general associations between the measures, we first ran correlation analyses between the four measures of social cognition (accuracy to infer the speaker's perspective in the interactive reference assignment task, accuracy to infer the mental states of others in the strange stories 
task, egocentric and altercentric bias in the visual perspective-taking task), loneliness, selfreported social functioning, and age. Next, we used multivariable regression models, separately for each social cognition measure, to establish whether loneliness is a predictor of social cognitive abilities independently of the other factors.

\subsection{Correlation Analyses}

A series of correlations examined relations between performance on the interactive reference assignment task, strange stories task, visual perspective-taking task (egocentric and altercentric bias), loneliness, social functioning, and age (see Table 1). A significant negative correlation emerged between loneliness and accuracy on the interactive reference assignment task, $r(96)=-.22, p=.032$, revealing that participants who self-reported higher levels of loneliness and feelings of social isolation were more likely to commit egocentric errors when taking the speaker's perspective. Similarly, a significant positive correlation emerged between loneliness and the egocentric bias on the visual perspective-taking task, $r(96)=.22$, $p=.032$, as people with higher levels of loneliness and feelings of social isolation showed a larger egocentric bias when taking the avatar's perspective. Loneliness also negatively correlated with social functioning, $r(96)=-.48, p<.001$, showing that older adults with higher levels of loneliness and feelings of social isolation were less likely to engage in social interaction and prosocial activities. In addition, loneliness significantly correlated with age, $r(96)=.27, p=.008$, as higher levels of loneliness and feelings of social isolation were reported with increasing age. Finally, age correlated significantly with accuracy on the interactive reference assignment task, $r(96)=-.35, p<.001$, and with egocentric bias on the visual perspective-taking task, showing that participants committed more egocentric errors when taking the speaker's perspective/adopting an avatar's visual perspective with advancing 
age. Performance on the strange stories task and the altercentric bias on the visual perspective-taking task did not correlate with any of the other measures (all $p_{s}>.17$ ).

\begin{tabular}{|l|l|l|l|l|l|l|}
\hline & 2. & 3. & 4. & 5. & 6. & 7. \\
\hline 1. Loneliness & $-.48^{* * *}$ & -.05 & $-.22^{*}$ & $.22^{*}$ & -.14 & $.27^{* *}$ \\
\hline 2. Social Functioning & & .11 & .06 & -.10 & .03 & -.14 \\
\hline 3. Strange Stories & & & .13 & -.04 & .05 & .02 \\
\hline 4. Reference Assignment & & & & -.05 & -.11 & $-.35^{* * *}$ \\
\hline 5. VPT Egocentric Bias & & & & & $.26^{* *}$ & $.28^{* *}$ \\
\hline 6. VPT Altercentric Bias & & & & & & .12 \\
\hline 7. Age & & & & & & \\
\hline
\end{tabular}

Table 1: Correlation matrix illustrating the relationships between performance on the interactive reference assignment task, strange stories task, visual perspective-taking task (egocentric and altercentric bias), loneliness, social functioning, and age. Asterisks indicate a significant correlation $(* p<.05, * * p<.01, * * * p<.001)$, and the cell values show $r$.

\subsection{Multivariable regression models}

A series of regression models were conducted, separately for the interactive reference assignment task, the strange stories task, and visual perspective-taking task (egocentric and altercentric bias) to test the degree to which performance on these social cognition measures can be explained by loneliness, social functioning, and age. The first models included loneliness as the independent variable and scores for social cognition measure as the dependent variables. In the second step, we added the social functioning score as a predictor to test whether any observed association between social cognition and loneliness was independent of self-reported social functioning. Finally, the third model added age as 
predictor. Statistical data are presented in Table 2, and regression plots are shown in the Supplementary Materials, Figure A. 
Table 2: Multivariable regression analysis on accuracy in the Interactive Reference Assignment and the Strange Stories task, where $* p<.05$, ** $p<$ .01

\begin{tabular}{|c|c|c|c|c|c|c|c|c|c|}
\hline & \multicolumn{3}{|c|}{ Model 1} & \multicolumn{3}{|c|}{ Model 2} & \multicolumn{3}{|c|}{ Model 3} \\
\hline & $\beta$ & $95 \% \mathrm{CI}$ & $p$ & $\beta$ & $95 \% \mathrm{CI}$ & $p$ & $\beta$ & $95 \% \mathrm{CI}$ & $p$ \\
\hline \multicolumn{10}{|c|}{ Interactive Reference Assignment } \\
\hline Loneliness & -.006 & $-.012,<-.001$ & $.032 *$ & -.007 & $-.014,<-.001$ & $.035^{*}$ & -.005 & $-.011, .002$ & .150 \\
\hline Social Functioning & & - & & -.002 & $-.008, .005$ & .623 & -.002 & $-.008, .004$ & .576 \\
\hline Age & & - & & & - & & -.013 & $-.021,-.005$ & $.002 * *$ \\
\hline$R^{2}$ & & $.047^{*}$ & & & .050 & & & $.142 * *$ & \\
\hline \multicolumn{10}{|l|}{ Strange Stories } \\
\hline Loneliness & -.011 & $-.057, .035$ & .635 & .001 & $-.051, .053$ & .966 & -.001 & $-.055, .052$ & .961 \\
\hline Social Functioning & & - & & .025 & $-.027, .077$ & .341 & .025 & $-.027, .077$ & .340 \\
\hline Age & & - & & & - & & .013 & $-.054, .081$ & .694 \\
\hline$R^{2}$ & & .002 & & & .012 & & & .014 & \\
\hline \multicolumn{10}{|c|}{ Visual perspective-taking, Egocentric bias } \\
\hline Loneliness & 4.60 & $.412,8.78$ & $.032 *$ & 4.59 & $-.205,9.39$ & .060 & 3.26 & $-1.55,8.06$ & .182 \\
\hline
\end{tabular}




\begin{tabular}{|c|c|c|c|c|c|c|c|c|}
\hline Social Functioning & - & & -.010 & $-4.76,4.74$ & .996 & .069 & $-4.57,4.71$ & .976 \\
\hline Age & - & & & - & & 7.30 & $1.27,13.33$ & $.018^{*}$ \\
\hline$R^{2}$ & $.047 *$ & & & .047 & & & $.102 *$ & \\
\hline \multicolumn{9}{|c|}{ Visual perspective-taking, Altercentric bias } \\
\hline Loneliness & $-5.82,1.05$ & .171 & -2.74 & $-6.67,1.20$ & .170 & -3.49 & $-7.50, .520$ & .087 \\
\hline Social Functioning & - & & -.728 & $-4.63,3.17$ & .711 & -.684 & $-4.55,3.18$ & .726 \\
\hline Age & - & & & - & & 4.10 & $-.925,9.13$ & .109 \\
\hline$\overline{R^{2}}$ & .019 & & & .021 & & & .047 & \\
\hline
\end{tabular}


For the interactive reference assignment task, regression analyses showed that higher levels of loneliness and feelings of social isolation were significantly related to increased egocentric errors when taking the speaker's perspective; this relation persisted after adjusting for social functioning. In these models, loneliness explained $\sim 5 \%$ of the variance in performance on the interactive reference assignment task. However, in model 3, where all three independent variables were entered into the regression model, loneliness was no longer statistically associated with performance on the interactive reference assignment task, but age showed a significant association. This model including age explained $14.2 \%$ of the variance, which suggests that the influence of loneliness in models 1 and 2 is relatively small, and is largely explained by the fact that people experience increasing levels of loneliness and feelings of social isolation with increasing age (as seen in the correlation analyses).

Similarly, regression analyses for the egocentric bias in the visual perspective-taking task showed that older adults with higher levels of loneliness and feelings of social isolation experienced greater egocentric interference when cued to take the avatar's visual perspective; the model explained $4.7 \%$ of the variance. However, when social functioning was added to the model, egocentric bias was no longer predicted by loneliness. Moreover, when age was added in model 3, only age predicted egocentric bias in the visual perspective-taking task, with this simultaneous model explaining $10.2 \%$ of variance. This pattern again suggests that while loneliness has a small effect on egocentric bias in visual perspective-taking, this is driven by the increasing levels of loneliness among older people.

None of the independent variables provided a reliable predictor of performance on the strange stories task, or influenced the strength of altercentric bias in the visual perspective-taking task. 


\section{Discussion}

Understanding others' mental states (i.e. their beliefs, emotions, intentions) and taking their perspectives has long been a topic of interest in psychology as these processes are key components of successful social interaction. Researchers have established theories on how these abilities develop, with a particular focus on infancy and early childhood (Apperly et al., 2010; Apperly \& Butterfil, 2009; Gregory et al., 2002). Interest in understanding how these processes change in older age has increased in recent years, with research generally reporting a ToM decline with advancing of age (Henry et al., 2013; Philips et al., 2011). Older age is often characterized by social isolation, which reduces the opportunities for social exchange and to exercise ToM processes, however very little research to date has empirically tested the relationship between loneliness and ToM in older age. In this paper we examined how social cognitive skills in older age are affected by subjective feelings of loneliness and social isolation. To our knowledge, this study is the first to adopt multiple measures of social cognitive abilities to investigate their relationship with loneliness in older age.

First, even within this older adult age range (60-86 years old), our results showed the expected influence of advancing age on social cognitive skills. As age increased, participants made more errors on the interactive reference assignment task and experienced greater egocentric interference on the visual perspective-taking task. This pattern fits with previous studies that have shown particular age-related impairments in the cognitive control mechanisms that underlie egocentric biases, as older adults have a self-biased processing style and experience difficulties ignoring and switching away from their own perspective (Bradford et al., submitted; Hultsch et al., 2002; Mattan et al., 2017; Martin et al., 2019; Saryazdi \& Chambers, 2020). Notably, these previous studies have examined effects of age by comparing dichotomous young and old groups, thus this study is the first show that the 
ability to control egocentric tendencies continues to decline throughout older age. In contrast, neither the strange stories task or the altercentric bias on the visual perspective-taking task correlated with age, which suggests that these processes are relatively automatically activated and not subject to age-related decline with decreasing cognitive resources (Apperly \& Butterfil, 2009; De Lillo \& Ferguson, submitted). Of course, it is also possible that mentalising and altercentric tendencies do change from young to older age, but do not continue to change once in older age. This would be consistent with the cubic pattern of change in Strange Stories performance across the lifespan reported by Brunsdon et al. (2019), where ToM scores increased from 10 years to 30 years of age, decreased from 30 to 70 years old then plateaued from 70 years of age onwards. Further research is needed to test this.

More importantly, our results revealed a small but significant relationship between loneliness and perspective-taking. Correlation analyses indicated that people who made more egocentric errors on the interactive reference assignment task and were more susceptible to egocentric interference on the visual perspective-taking task were more likely to self-report higher levels of loneliness and feelings of social isolation. These relationships were further confirmed by the multivariable regression analysis, which showed that loneliness predicted accuracy on the interactive reference assignment task and egocentric interference on the visual perspectivetaking task. However, this relationship between loneliness and egocentric perspective-taking tendencies did not persist when age was added to the models, which suggests that people experience increasing levels of loneliness and feelings of social isolation with increasing age, but loneliness and ToM are not related once advancing age is controlled for. Cacioppo and Hawkley (2009) have suggested that loneliness might increase egocentric tendencies by inducing higher sensitivity to social threats, which biases peoples' expectations for and memories of negative social interactions. As a result, individuals become more likely to 
experience negative social interactions, therefore altering the nature and likelihood of social engagement. Considered in the context of aging, it is therefore possible that declining ToM skills in older age, and reduced opportunities to interact with others, increases the difficulty that older adults experience with social interaction (e.g. more miscommunications), which leads them to withdraw from social situations and become more lonely. Future studies should examine more closely how these cognitive and affective processes interact with each other, including the direction of these relationships, and how they are manifest across different subcomponents of ToM.

In contrast, no association was found between loneliness and mentalizing abilities in the strange stories task or the altercentric bias in the visual-perspective-taking task. This suggests that the impact of loneliness on social cognition is likely to operate via the underlying cognitive mechanisms that enable people to inhibit their own egocentric perspective and flexibly shift between self and other perspectives, and these are known to decline significantly in older age and predict social interaction skills (e.g. Phillips et al., 2011, 2015). These findings are also consistent with previous research that has shown preserved or even enhanced sensitivity to others' mental states among older adults compared to younger adults (Hess et al., 2005), and that this sensitivity does not relate to basic cognitive skills (Leclerc \& Hess, 2007), with these effects explained in terms of accumulating experience in social situations over the lifecourse.

In line with the effects of loneliness and age on egocentric perspective-taking described above, we found that loneliness correlated with age, confirming existing research that people experience increasing feelings of loneliness with advancing age (Richard et al., 2017; Yang \& Victor, 2011), particularly after the age of 75 years old (Ferreira-Alves et al., 2014). It is 
interesting to note that age did not correlate with social functioning in our older adults. This likely reflects the nature of our participant sample, who were sufficiently motivated to volunteer their time to take part and were often recruited through networks of older adults (e.g. the University of the Third Age). As such, the older adults here may not be representative of the breadth of individuals in the general public who are more socially isolated and have reduced mobility. Nevertheless, as a group, older adults with higher levels of loneliness and negative feelings of social isolation reported lower levels of social functioning- they were less likely to engage in social interaction and prosocial activitieswhich suggests that our sample included sufficient variance in loneliness to capture the relationship with social functioning. This finding is consistent with a recent study (Tan et al., 2020) that found higher levels of loneliness among adults who reported reduced enjoyment in social activities, suggesting that they function less well in social situations.

In sum, the current research revealed that loneliness plays a role in older adults' ability to manage egocentric biases in perspective-taking, but does not relate with the ability to infer other peoples' mental states and perspectives. Importantly, this study showed that the effects of loneliness on social cognition in older age are eliminated once participant age is controlled for. Therefore, while there may be a small influence of loneliness on egocentric control, this is explained by the fact that people experience increasing levels of loneliness and feelings of social isolation with increasing age. Based on evidence that people who experience loneliness engage less in group activities because of their inadequate social skills (Page et al., 1992; Pels \& Kleinert, 2016) and lack self-regulation in social situations (Hawkley et al., 2009; Netz et al., 2013), it would be useful for future research to include measures that assess quality of life, and account for social network size, since these are known to be related to age, loneliness and ToM abilities. Understanding these relations better, ideally using a 
longitudinal approach to identify precursors and predictive to social cognitive difficulties, could help to develop more targeted interventions to enhance well-being and the quality of social relationships, particularly among older adults.

\section{References}

Apperly, I. (2010). Mindreaders: the cognitive basis of" theory of mind". Psychology Press.

Apperly, I. A., \& Butterfill, S. A. (2009). Do humans have two systems to track beliefs and belief-like states? Psychological review, 116(4), 953.

Bailey, P. E., Henry, J. D., \& Von Hippel, W. (2008). Empathy and social functioning in late adulthood. Aging and Mental Health, 12(4), 499-503.

Berkman, L. F. (1995). The role of social relations in health promotion. Psychosomatic medicine, 57(3), 245-254

Berkman, L. F., Glass, T., Brissette, I., \& Seeman, T. E. (2000). From social integration to health: Durkheim in the new millennium. Social science \& medicine, 51(6), 843-857.

Bernstein, D. M., Thornton, W. L., \& Sommerville, J. A. (2011). Theory of mind through the ages: Older and middle-aged adults exhibit more errors than do younger adults on a continuous false belief task. Experimental Aging Research, 37(5), 481-502.

Birchwood, M., Smith, J. O., Cochrane, R., Wetton, S., \& Copestake, S. O. N. J. A. (1990). The social functioning scale the development and validation of a new scale of social adjustment for use in family intervention programmes with schizophrenic patients. The British Journal of Psychiatry, 157(6), 853-859.

Boss, L., Kang, D. H., \& Branson, S. (2015). Loneliness and cognitive function in the older adult: a systematic review. International Psychogeriatrics, 27(4), 541. 
Bradford, E. F.., Brunsdon, V. E. A., \& Ferguson, H. J. (submitted). Cognitive Mechanisms of Perspective-Taking Across Adulthood: An Eye-Tracking Study Using the Director Task.

Brunsdon, V. E. A., Bradford, E. F., \& Ferguson, H. J. (2019). Sensorimotor mu rhythm during action observation changes across the lifespan independently from social cognitive processes. Developmental cognitive neuroscience, 38, 100659.

Cacioppo, J. T., \& Hawkley, L. C. (2009). Perceived social isolation and cognition. Trends in cognitive sciences, 13(10), 447-454.

Cacioppo, J. T., Hughes, M. E., Waite, L. J., Hawkley, L. C., \& Thisted, R. A. (2006). Loneliness as a specific risk factor for depressive symptoms: cross-sectional and longitudinal analyses. Psychology and aging, 21(1), 140.

Carson, J., Prescott, J., Allen, R., and McHugh, S. (2020). Winter is coming: age and early psychological concomitants of the Covid-19 pandemic in England. J. Public Ment. Health 19, 221-230. doi: 10.1108/JPMH-06-2020-0062

Cavallini, E., Lecce, S., Bottiroli, S., Palladino, P., \& Pagnin, A. (2013). Beyond false belief: Theory of mind in young, young-old, and old-old adults. International Journal of Aging \& Human Development, 76, 181-198.

De Lillo, M. \& Ferguson H. J., (submitted) Perspective-taking and social inferences in adolescents, young adults and older adults.

Devine, R. T., \& Hughes, C. (2013). Silent films and strange stories: Theory of mind, gender, and social experiences in middle childhood. Child development, 84(3), 989-1003.

Diener, E., \& Seligman, M. E. (2002). Very happy people. Psychological science, 13(1), 8184. 
Domènech-Abella, J., Lara, E., Rubio-Valera, M., Olaya, B., Moneta, M. V., Rico-Uribe, L. A., ... \& Haro, J. M. (2017). Loneliness and depression in the elderly: the role of social network. Social psychiatry and psychiatric epidemiology, 52(4), 381-390.

Dumontheil, I., Apperly, I. A., \& Blakemore, S. J. (2010). Online usage of theory of mind continues to develop in late adolescence. Developmental science, 13(2), 331-338.

Ferguson, H. J., Apperly, I., \& Cane, J. E. (2017). Eye tracking reveals the cost of switching between self and other perspectives in a visual perspective-taking task. Quarterly Journal of Experimental Psychology, 70(8), 1646-1660.

Ferreira-Alves, J., Magalhães, P., Viola, L., \& Simoes, R. (2014). Loneliness in middle and old age: Demographics, perceived health, and social satisfaction as predictors. Archives of gerontology and geriatrics, 59(3), 613-623.

Flavell, J. H., Green, F. L., and Flavell, E. R. (1986). Development of knowledge about the appearance-reality distinc-tion. Monogr. Soc. Res. Child Dev. 51, i-v, 1-87.

Gregory, C., Lough, S., Stone, V., Erzinclioglu, S., Martin, L., Baron-Cohen, S., \& Hodges, J. R. (2002). Theory of mind in patients with frontal variant frontotemporal dementia and Alzheimer's disease: theoretical and practical implications. Brain, 125(4), 752764.

Halberstadt, J., Ruffman, T., Murray, J., Taumoepeau, M., \& Ryan, M. (2011). Emotion perception explains age-related differences in the perception of social gaffes. Psychology and Aging, 26(1), 133.

Happé, F. G., Winner, E., \& Brownell, H. (1998). The getting of wisdom: theory of mind in old age. Developmental psychology, 34(2), 358.

Hawkley, L. C., \& Cacioppo, J. T. (2010). Loneliness matters: A theoretical and empirical review of consequences and mechanisms. Annals of behavioral medicine, 40(2), 218227. 
Hawkley, L. C., \& Capitanio, J. P. (2015). Perceived social isolation, evolutionary fitness and health outcomes: a lifespan approach. Philosophical Transactions of the Royal Society B: Biological Sciences, 370(1669), 20140114.

Hawkley, L. C., Thisted, R. A., \& Cacioppo, J. T. (2009). Loneliness predicts reduced physical activity: Cross-sectional \& longitudinal analyses. Health Psychology, 28(3), 354-363. doi:10.1037/a0014400

Heid, A. R., Cartwright, F., Wilson-Genderson, M., and Pruchno, R. (2021). Challenges experienced by older people During the initial months of the COVID-19 pandemic. The Gerontologist 61, 48-58. doi: 10.1093/geront/gnaa138

Heidinger, T., \& Richter, L. (2020). The effect of COVID-19 on loneliness in the elderly. An empirical comparison of pre-and peri-pandemic loneliness in community-dwelling elderly. Frontiers in Psychology, 11, 2595.

Henry, J. D., Phillips, L. H., Ruffman, T., \& Bailey, P. E. (2013). A meta-analytic review of age differences in theory of mind. Psychology and Aging, 28(3), 826.

Hess, T. M., Osowski, N. L., \& Leclerc, C. M. (2005). Age and experience influences on the complexity of social inferences. Psychology and aging, 20(3), 447.

Hultsch, D. F., MacDonald, S. W., \& Dixon, R. A. (2002). Variability in reaction time performance of younger and older adults. The Journals of Gerontology Series B: Psychological Sciences and Social Sciences, 57(2), P101-P115.

Keysar, B., Lin, S., \& Barr, D. J. (2003). Limits on theory of mind use in adults. Cognition, 89(1), 25-41.

Lecce, S., Ceccato, I., Bianco, F., Rosi, A., Bottiroli, S., \& Cavallini, E. (2017). Theory of Mind and social relationships in older adults: the role of social motivation. Aging \& Mental Health, 21(3), 253-258. 
Lecce, S., Ceccato, I., \& Cavallini, E. (2019). Investigating ToM in aging with the MASC: from accuracy to error type. Aging, Neuropsychology, and Cognition, 26(4), 541-557.

Lecce, S., Zocchi, S., Pagnin, A., Palladino, P., \& Taumoepeau, M. (2010). Reading minds: The relation between children's mental state knowledge and their metaknowledge about reading. Child Development, 81(6), 1876-1893.

Leclerc, C. M., \& Hess, T. M. (2007). Age differences in the bases for social judgments: Tests of a social expertise perspective. Experimental Aging Research, 33(1), 95-120.

Leslie, A. M. (2005). Developmental parallels in understanding minds and bodies. Trends in Cognitive Sciences, 9(10), 459-462.

Lim, M. H., \& Gleeson, J. F. (2014). Social connectedness across the psychosis spectrum: current issues and future directions for interventions in loneliness. Frontiers in psychiatry, 5, 154.

Lim, M. H., Gleeson, J. F., Alvarez-Jimenez, M., \& Penn, D. L. (2018). Loneliness in psychosis: a systematic review. Social psychiatry and psychiatric epidemiology, 53(3), 221-238.

Lim, M. H., Rodebaugh, T. L., Zyphur, M. J., \& Gleeson, J. F. (2016). Loneliness over time: The crucial role of social anxiety. Journal of abnormal psychology, 125(5), 620.

Lund, C., Standsfield, S., \& De Silva, M. (2013). Social determinants of mental disorders. Global Mental Health: Principles and Practice.

Martin, A. K., Perceval, G., Davies, I., Su, P., Huang, J., \& Meinzer, M. (2019). Visual perspective taking in young and older adults. Journal of Experimental Psychology: General, 148(11), 2006.

Matheson, J. (2011). Measuring what matters: National statistician's reflections on the national debate on measuring national well-being. UK Office for National Statistics. Retrieved from http://www.ons.gov.uk/ons/guide-method/user-guidance/well- 
being/publications/measuring-what-matters-national-statistician-s-reflections-on-thenational-debate-on-measuringnational-well-being.pdf

Mattan, B. D., Quinn, K. A., Acaster, S. L., Jennings, R. M., \& Rotshtein, P. (2017). Prioritization of self-relevant perspectives in ageing. Quarterly Journal of Experimental Psychology, 70(6), 1033-1052.

Maylor, E. A., Moulson, J. M., Muncer, A. M., \& Taylor, L. A. (2002). Does performance on theory of mind tasks decline in old age? British Journal of Psychology, 93(4), 465485.

Meltzer, H., Bebbington, P., Dennis, M. S., Jenkins, R., McManus, S., \& Brugha, T. S. (2013). Feelings of loneliness among adults with mental disorder. Social psychiatry and psychiatric epidemiology, 48(1), 5-13.

Nangle, D. W., Erdley, C. A., Newman, J. E., Mason, C. A., \& Carpenter, E. M. (2003). Popularity, friendship quantity, and friendship quality: Interactive influences on children's loneliness and depression. Journal of Clinical Child and Adolescent Psychology, 32(4), 546-555.

National Academies of Sciences, Engineering, and Medicine. 2020. Social Isolation and Loneliness in Older Adults: Opportunities for the Health Care System. Washington, DC: The National Academies Press. https://doi.org/10.17226/25663external icon

Netz, Y., Goldsmith, R., Shimony, T., Arnon, M., \& Zeev, A. (2013). Loneliness is associated with an increased risk of sedentary life in older Israelis. Aging \& Mental Health, 17(1), 40-47. doi:10. 1080/13607863.2012.715140

Page, R. M., Frey, J., Talbert, R., \& Falk, C. (1992). Children's feelings of loneliness and social dissatisfaction: Relationship measures of physical fitness and activity. Journal of Teaching in Physical Education, 11, 211-219. 
Pels, F., \& Kleinert, J. (2016). Loneliness and physical activity: A systematic review. International Review of Sport and Exercise Psychology, 9(1), 231-260.

Peng, S., and Roth, A. R. (2021). Social isolation and loneliness Before and During the COVID-19 pandemic: A longitudinal study of U.S. adults older Than 50. J. Gerontol. Series B 69, 20-29. doi: 10.1093/geronb/gbab068

Phillips, L. H., Allen, R., Bull, R., Hering, A., Kliegel, M., \& Channon, S. (2015). Older adults have difficulty in decoding sarcasm. Developmental psychology, 51(12), 1840.

Phillips, L. H., Bull, R., Allen, R., Insch, P., Burr, K., \& Ogg, W. (2011). Lifespan aging and belief reasoning: Influences of executive function and social cue decoding. Cognition, 120(2), 236-247.

Pinquart, M., \& Sorensen, S. (2001). Influences on loneliness in older adults: A metaanalysis. Basic and applied social psychology, 23(4), 245-266.

Richard, A., Rohrmann, S., Vandeleur, C. L., Schmid, M., Barth, J., \& Eichholzer, M. (2017). Loneliness is adversely associated with physical and mental health and lifestyle factors: Results from a Swiss national survey. PloS one, 12(7), e0181442.

Russell, D. W. (1996). UCLA Loneliness Scale (Version 3): Reliability, validity, and factor structure. Journal of personality assessment, 66(1), 20-40.

Samson, D., Apperly, I. A., Braithwaite, J. J., Andrews, B. J., \& Bodley Scott, S. E. (2010). Seeing it their way: evidence for rapid and involuntary computation of what other people see. Journal of Experimental Psychology: Human Perception and Performance, $36(5), 1255$.

Santamaría-García, H., Baez, S., Gómez, C., Rodríguez-Villagra, O., Huepe, D., Portela, M., ... \& Ibanez, A. (2020). The role of social cognition skills and social determinants of health in predicting symptoms of mental illness. Translational psychiatry, 10(1), 1-13. 
Saryazdi R, Chambers CG. Real-time communicative perspective taking in younger and older adults. J Exp Psychol Learn Mem Cogn. 2020 Oct 1. doi: 10.1037/xlm0000890. Epub ahead of print. PMID: 33001700.

Shankar, A., Rafnsson, S. B., \& Steptoe, A. (2015). Longitudinal associations between social connections and subjective wellbeing in the English Longitudinal Study of Ageing. Psychology \& health, 30(6), 686-698.

Spitzberg, B. H., \& Hurt, H. T. (1987). The relationship of interpersonal competence and skills to reported loneliness across time. Journal of Social Behavior and Personality, 2(2), 157.

Stiller, J., \& Dunbar, R. I. (2007). Perspective-taking and memory capacity predict social network size. Social Networks, 29(1), 93-104.

Symeonidou, I., Ferguson, H. J., Dumontheil, I., \& Breheny, R. (2020). Author accepted manuscript: Adolescents are delayed at inferring complex social intentions in others, but not basic (false) beliefs: An eye movement investigation. Quarterly Journal of Experimental Psychology, 1747021820920213.

Tan, M., Shallis, A., \& Barkus, E. (2020). Social anhedonia and social functioning: Loneliness as a mediator. PsyCh journal, 9(2), 280-289.

Tilvis, R. S., Kähönen-Väre, M. H., Jolkkonen, J., Valvanne, J., Pitkala, K. H., \& Strandberg, T. E. (2004). Predictors of cognitive decline and mortality of aged people over a 10year period. The Journals of Gerontology Series A: Biological Sciences and Medical Sciences, 59(3), M268-M274.

Trémeau, F., Antonius, D., Malaspina, D., Goff, D. C., \& Javitt, D. C. (2016). Loneliness in schizophrenia and its possible correlates. An exploratory study. Psychiatry research, 246, 211-217. 
Tsai, F. F., \& Reis, H. T. (2009). Perceptions by and of lonely people in social networks. Personal Relationships, 16(2), 221-238.

Valtorta, N. K., Kanaan, M., Gilbody, S., Ronzi, S., \& Hanratty, B. (2016). Loneliness and social isolation as risk factors for coronary heart disease and stroke: systematic review and meta-analysis of longitudinal observational studies. Heart, 102(13), 1009-1016.

White, S., Hill, E., Happé, F., \& Frith, U. (2009). Revisiting the strange stories: Revealing mentalizing impairments in autism. Child development, 80(4), 1097-1117.

Wilson, R. S., Krueger, K. R., Arnold, S. E., Schneider, J. A., Kelly, J. F., Barnes, L. L., ... \& Bennett, D. A. (2007). Loneliness and risk of Alzheimer disease. Archives of general psychiatry, 64(2), 234-240.

Wimmer, H., \& Perner, J. (1983). Beliefs about beliefs: Representation and constraining function of wrong beliefs in young children's understanding of deception. Cognition, $13,103-128$.

Yang, K., \& Victor, C. (2011). Age and loneliness in 25 European nations. Ageing and Society, 31(8), 1368. 